\title{
INTRINSIC HARDY-ORLICZ SPACES OF CONFORMAL MAPPINGS
}

\author{
PEKKA KOSKELA AND SITA BENEDICT
}

\begin{abstract}
We define a new type of Hardy-Orlicz spaces of conformal mappings on the unit disk where in place of the value $|f(x)|$ we consider the intrinsic path distance between $f(x)$ and $f(0)$ in the image domain. We show that if the Orlicz function is doubling then these two spaces are actually the same, and we give an example when the intrinsic Hardy-Orlicz space is strictly smaller.
\end{abstract}

\section{INTRODUCTION}

Let $\psi:[0, \infty] \rightarrow[0, \infty]$ be a differentiable and strictly increasing function such that $\psi(0)=0$; that is, a growth function. A conformal map $f: \mathbb{D} \rightarrow \mathbb{C}$ belongs to the Hardy-Orlicz space $H^{\psi}$ if there exists $\delta>0$ such that

$$
\sup _{0<r<1} \int_{\partial \mathbb{D}} \psi(\delta|f(r \omega)|) d \sigma<\infty
$$

and to $H^{\infty}$ if

$$
\sup _{0<r<1} M(r, f)<\infty
$$

where

$$
M(r, f)=\sup _{\omega \in \partial \mathbb{D}}\{|f(r \omega)|\}
$$

is the maximum modulus function on $0<r<1$. Some results on Hardy-Orlicz spaces can be found in [2], [3], [6], [7, [11], and [13, however the exact definition of the spaces varies and the theory is not limited to conformal mappings on $\mathbb{D}$. In the case $\psi(t)=$ $t^{p}, 0<p<\infty$, see 8 .

Since a conformal map induces a change of metric in $\mathbb{D}$, the intrinsic path distance $d_{I}(f(x), f(0))$ in $f(\Omega)$ is in many occasions more natural than $|f(x)|$, see for example [4]. We abbreviate $|f(x)|_{I}=d_{I}(f(x), f(0))$ and say that $f$ belongs to the intrinsic HardyOrlicz space $H_{I}^{\psi}$ if there exists $\delta>0$ such that

$$
\sup _{0<r<1} \int_{\partial \mathbb{D}} \psi\left(\delta|f(r \omega)|_{I}\right) d \sigma<\infty
$$

and to $H_{I}^{\infty}$ if $|f(x)|_{I}$ is uniformally bounded on $\mathbb{D}$. Here $d \sigma$ denotes integration with respect to the length measure on $\partial \mathbb{D}$.

If $f(\mathbb{D})$ is a bounded domain then $f \in H^{\infty}$ by definition. It is easy to see that there are conformal mappings that belong to $H^{\infty}$ but do not belong to $H_{I}^{\infty}$; one only has to consider a conformal mapping of the unit disk onto a bounded domain that spirals inward ad infinitum. For general $\psi$ it is not obvious whether there are conformal mappings belonging to $H^{\psi}$ but not to $H_{I}^{\psi}$. In this paper we show somewhat surprisingly that if $\psi$ is doubling then there are no such mappings.

\footnotetext{
2010 Mathematics Subject Classification: 30C35, 30H10

Key words and phrases. Hardy spaces, Hardy-Orlicz, conformal mappings on the unit disk

The authors were partially supported by the Academy of Finland grants 131477 and 263850.
} 
Theorem 1.1. Let $f$ be a conformal mapping of $\mathbb{D}$. If $\psi$ is a doubling growth function then $f \in H^{\psi}$ if and only if $f \in H_{I}^{\psi}$.

Thus, our theorem gives a new characterization of the conformal mappings belonging to the classical $H^{p}$ spaces for all $0<p<\infty$. Using the Gehring-Hayman inequality we also obtain the following.

Corollary 1.2. Let $f$ be a conformal mapping of $\mathbb{D}$ and $\psi$ a doubling growth function. Then

$$
f \in H^{\psi} \quad \text { if and only if } \int_{\partial \mathbb{D}} \psi\left(\int_{0}^{1}\left|f^{\prime}(r \omega)\right| d r\right) d \sigma<\infty
$$

We will give examples to show that if the $\psi$ is not doubling then the result may fail, see Section 5.

The authors would like to dedicate this paper to both Kari Astala and Michel Zinsmeister, whose ideas in [1] and [15] respectively have contributed to several of the techniques used in this paper.

\section{BAsic Definitions}

Denote by $\mathbb{D}=\{x \in \mathbb{C}:|x|<1\}$ the open unit disk in the complex plane, by $\partial \mathbb{D}$ its boundary the unit circle, by $B(x, r)$ the open disk centered at $x \in \mathbb{C}$ with radius $r>0$ and by $\partial B(x, r)$ its boundary. An analytic function $f: \Omega \rightarrow \mathbb{C}$ on a domain $\Omega \subset \mathbb{C}$ is called conformal if it is also injective. In this paper the conformal mappings we consider will all have as domain $\mathbb{D}$.

A curve in a domain $\Omega \subset \mathbb{C}$ is a continuous mapping $\gamma: I \rightarrow \Omega$ of an interval $I \subseteq \mathbb{R}$. The image set $\gamma(I)$ is also denoted by $\gamma$. The euclidean length of the curve is denoted by length $(\gamma) \in[0, \infty]$. If length $(\gamma)<\infty$ then $\gamma$ is rectifiable. A curve is called locally rectifiable if length $\left(\left.\gamma\right|_{[a, b]}\right)<\infty$ for every closed sub-interval $[a, b] \subset I$. We allow that the endpoints of a curve in $\mathbb{D}$ may belong to $\partial \mathbb{D}$.

Given a simply connected domain $\Omega$ strictly contained in $\mathbb{C}$, the Riemann Mapping Theorem asserts the existence of a conformal function $f$ mapping $\mathbb{D}$ onto $\Omega$. Recall that if $\gamma$ is a curve in $\mathbb{D}$ then $f \circ \gamma$ is a curve in $\Omega$, and when integrating with respect to arc length we have

$$
\operatorname{length}(f \circ \gamma)=\int_{\gamma}\left|f^{\prime}(z)\right||d z| .
$$

Given $u, v \in \Omega$ the intrinsic path distance between $u$ and $v$ in $\Omega$ is

$$
d_{I}(u, v):=\inf \operatorname{length}(\gamma)
$$

where the infimum is taken over all curves $\gamma$ in $\Omega$ with endpoints $u$ and $v$. This defines a metric on $\Omega$. If $f$ maps $\mathbb{D}$ conformally onto $\Omega$ and $x=f^{-1}(u)$ and $y=f^{-1}(v)$ then clearly

$$
d_{I}(u, v)=\inf \operatorname{length}(f \circ \gamma),
$$

where the infimum is taken over all curves in $\mathbb{D}$ with endpoints $x$ and $y$. We abbreviate then $d_{I}(f(x), f(0))$ to $|f(x)|_{I}$ for each $x \in \mathbb{D}$, and we denote usual metric space notions with respect to $d_{I}$ with the addition of the subscript ${ }_{I}$. For instance, the diameter of a set $E \subset \Omega$ in the metric $d_{I}$ will be denoted by $\operatorname{diam}_{I}(E)$.

It is well known that if $f$ is a conformal mapping of $\mathbb{D}$ then the radial limit

$$
\lim _{r \rightarrow 1} f(r \omega)=f(\omega)
$$


exists and length $(f([0, \omega))<\infty$ for almost every $\omega \in \partial \mathbb{D}$, see for example [12]. If $f(\omega)$ exists we define for each $x \in \mathbb{D}$ the intrinsic path distance

$$
d_{I}(f(x), f(\omega))=\inf \text { length }(f \circ \gamma),
$$

where the infimum is taken over all curves $\gamma$ in $\mathbb{D}$ with endpoints $x$ and $\omega$, and we abbreviate like before

$$
|f(\omega)|_{I}:=d_{I}(f(0), f(\omega)) .
$$

We will mostly be maneuvering through $\mathbb{D}$ via 'Whitney-type disks,' their corresponding 'shadows' on $\partial \mathbb{D}$ as well as the related Stolz cones, which we define here. Given $x \in \mathbb{D}$ let the Whitney disk centered at $x$ be defined as $B_{x}=B\left(x, \frac{1-|x|}{2}\right)$, and let $S_{x}=\left\{\frac{z}{|z|}: z \in B_{x}\right\}$ denote its shadow on $\partial \mathbb{D}$. For each $\omega \in \partial \mathbb{D}$ let

$$
\Gamma(\omega)=\bigcup\left\{B_{t \omega}: 0 \leq t<1\right\}
$$

be the Stolz cone at $\omega$. We associate with each conformal mapping $f: \mathbb{D} \rightarrow \mathbb{C}$ its non-tangential maximal function with respect to the euclidean metric as

$$
f^{*}(\omega)=\sup _{x \in \Gamma(\omega)}|f(x)|, \omega \in \partial \mathbb{D},
$$

and its non-tangential maximal function with respect to $d_{I}$ as

$$
f_{I}^{*}(\omega)=\sup _{x \in \Gamma(\omega)}|f(x)|_{I}, \omega \in \partial \mathbb{D} .
$$

\section{Koebe, Modulus estimates, and Gehring-Hayman}

We begin this section by stating two corollaries to the well-known Koebe distortion theorem, see [12, Corollaries 1.4 and 1.5].

Lemma 3.1. There exists a universal constant $C$ such that if $f$ is a conformal mapping of $\mathbb{D}$ then

$$
\frac{1}{C} d(f(x), \partial f(\mathbb{D})) \leq\left|f^{\prime}(x)\right|(1-|x|) \leq C d(f(x), \partial f(\mathbb{D}))
$$

for all $x \in \mathbb{D}$.

The notation $d_{h}(x, y)$ in the next statement denotes the hyperbolic metric on $\mathbb{D}$.

Lemma 3.2. If $f: \mathbb{D} \rightarrow \mathbb{C}$ is conformal then

$$
e^{-6 d_{h}(x, y)} \leq \frac{\left|f^{\prime}(x)\right|}{\left|f^{\prime}(y)\right|} \leq e^{6 d_{h}(x, y)}
$$

for any $x, y \in \mathbb{D}$.

This lemma tells us that $\left|f^{\prime}\right|$ is roughly constant on Whitney disks $B_{x}=B\left(x, \frac{1-|x|}{2}\right)$, $x \in \mathbb{D}$. Indeed if $x, y \in B_{z}$ for some $z \in \mathbb{D}$ then $d_{h}(x, y) \leq 2$, and so by setting $C=e^{12}$ we have

$$
\frac{1}{C} \leq \frac{\left|f^{\prime}(x)\right|}{\left|f^{\prime}(y)\right|} \leq C \text { for all } x, y \in B_{z}, z \in \mathbb{D} .
$$

It follows easily that there is an absolute constant $C$ such that

$$
\operatorname{diam}\left(f\left(B_{x}\right)\right) \leq \operatorname{diam}_{I}\left(f\left(B_{x}\right)\right) \leq C d(f(x), \partial f(\mathbb{D}))
$$

for all $x \in \mathbb{D}$. 
We collect some basic facts about modulus of curve families, needed for the lemmas that follow. If $\Gamma$ is a family of locally rectifiable curves in a domain $\Omega \subseteq \mathbb{C}$, a Borel function $\rho: \Omega \rightarrow[0, \infty]$ is called admissible if

$$
\int_{\gamma} \rho d s \geq 1
$$

for every curve $\gamma \in \Gamma$. The modulus $\operatorname{Mod}(\Gamma) \in[0, \infty]$ of the curve family $\Gamma$ is then defined as

$$
\inf \int_{\Omega} \rho^{2} d m
$$

where the infimum is taken over all admissible $\rho$. Here $d m$ denotes integration with respect to Lebesgue measure in the plane.

An important property of the modulus of curve families is that it is a conformal invariant. That is, when $f: \Omega \rightarrow \Omega^{\prime}$ is a conformal map between domains and $\Gamma$ is a family of curves in $\Omega$, then

$$
\operatorname{Mod}(\Gamma)=\operatorname{Mod}(f \Gamma)
$$

where $f \Gamma=\{f \circ \gamma: \gamma \in \Gamma\}$.

It is possible to compute the modulus or at least arrive at a useful estimate for certain families of curves. If $E$ is a Borel set in $\partial \mathbb{D}, 0<r<1$, and $\Gamma$ is the family of radial segments joining $\partial B(0, r)$ to $E$ then

$$
\operatorname{Mod}(\Gamma)=\frac{\sigma(E)}{(\log 1 / r)}
$$

More generally, the upper bound

$$
\operatorname{Mod}(\Gamma) \leq \frac{\sigma(\partial \mathbb{D})}{\log (R / r)}
$$

is valid whenever each $\gamma \in \Gamma$ joins $\partial B(x, r)$ to $\partial B(x, R), 0<r<R$. These and other basic properties of the modulus can be found in [14].

The next lemma is simply a special case of [4, Lemma 3.2], and so we omit its proof here.

Lemma 3.3. There exists a universal constant $C$ with the following property. Let $\Omega$ be a simply connected domain in $\mathbb{C}$ equipped with the intrinsic metric $d_{I}$, E a non-empty subset of $\Omega$ and suppose $L \geq \delta>0$. Assume that $\operatorname{diam}_{I}(E) \leq \delta$ and that $\Gamma$ is a family of curves in $\Omega$ so that each curve $\gamma \in \Gamma$ has one endpoint in $E$ and length $(\gamma) \geq L$. Then

$$
\operatorname{Mod}(\Gamma) \leq \frac{C}{\log (1+L / \delta)}
$$

The next two lemmas are used in Section 4 for our main result. Their proofs are similar, using basic modulus of curve families techniques.

Lemma 3.4. Let $f$ be a conformal mapping of $\mathbb{D}$ and $M>1$. There exists an absolute constant $C$ such that

$$
\sigma\left(\left\{\omega \in S_{x}: d_{I}(f(\omega), f(x))>M d(f(x), \partial f(\mathbb{D}))\right\}\right) \leq C \sigma\left(S_{x}\right)(\log M)^{-1}
$$

for every $x \in \mathbb{D}$. 
Proof. Fix $x \in \mathbb{D}$ and set $d=d(f(x), \partial f(\mathbb{D}))$ and $E=\left\{\omega \in S_{x}: d_{I}(f(\omega), f(x))>M d\right\}$. First suppose $|x|<1 / 4$ and let $\Gamma_{E}$ be the set of radial segments that have one endpoint in $E$ and the other in $B_{x} \cap \partial B(0,1 / 4)$. By (3.3) and the definition of $E$ each curve in $f\left(\Gamma_{E}\right)$ has one endpoint in $B_{I}(f(x), C d)$, where $C$ is absolute, and the other other endpoint in $\mathbb{C} \backslash B_{I}(f(x), M d)$. Assume $2 \leq C$ and $C^{2}<M$. Then by basic modulus estimates and Lemma 3.3 we have

$$
\sigma(E)(\log 4)^{-1}=\operatorname{Mod}\left(\Gamma_{E}\right)=\operatorname{Mod}\left(f\left(\Gamma_{E}\right)\right) \leq C(\log M)^{-1} \leq C \sigma(\partial \mathbb{D})(\log M)^{-1} .
$$

If $1<M \leq C^{2}$ then trivially

$$
\sigma(E) \leq \sigma(\partial \mathbb{D})\left(\log C^{2}\right)(\log M)^{-1} .
$$

If $|x| \geq 1 / 4$ then we choose $\Gamma_{E}$ to be the family of radial segments with one endpoint in $E$ and the other in $B_{x} \cap \partial B(0,|x|)$. Proceeding like before, the case $1<M \leq C^{2}$ is trivial and if $C^{2}<M$ then

$$
\sigma(E) \log (1 /|x|)^{-1}=\operatorname{Mod}\left(\Gamma_{E}\right)=\operatorname{Mod}\left(f\left(\Gamma_{E}\right)\right) \leq C(\log M)^{-1} .
$$

Noting that in this case $\log (1 /|x|) \approx \sigma\left(S_{x}\right)$, we are done.

Lemma 3.5. Let $f: \mathbb{D} \rightarrow \mathbb{C}$ be conformal map such that $f(x) \neq 0$ for all $x \in \mathbb{D}, \phi a$ growth function and $\delta>0$. There is an absolute constant $C$ such that for each $x \in \mathbb{D}$ and $M>1$,

$$
\sigma\left(\left\{\omega \in S_{x}: \phi(\delta|f(\omega)|)<\phi(\delta|f(x)| / M)\right\}\right) \leq C \sigma\left(S_{x}\right)(\log M)^{-1} .
$$

Proof. Let $x \in \mathbb{D}$ and $\omega \in S_{x}$. Since $\phi$ is strictly increasing, $\phi(\delta|f(\omega)|)<\phi(\delta|f(x)| / M)$ if and only if $|f(\omega)|<|f(x)| / M$. So it suffices to prove the inequality for the case that $\delta=1$ and $\phi$ is the identity map on $[0, \infty]$.

Set $E=\left\{\omega \in S_{x}:|f(\omega)|<|f(x)| / M\right\}$, and choose the curve families $\Gamma_{E}$ like in the proof of Lemma 3.4. Note that each curve in $f\left(\Gamma_{E}\right)$ will have one endpoint belonging to $B(f(x), C|f(x)|)$ and the other endpoint in $\mathbb{C} \backslash B(f(x),|f(x)| / M)$, with $C$ an absolute constant. The desired estimate then follows using the same properties of modulus of curve families as in the proof of Lemma 3.4.

We end this section with the following result due to Gehring and Hayman ([10]). Their theorem says that if $f: \mathbb{D} \rightarrow f(\mathbb{D})$ is conformal then the images of the hyperbolic geodesics in $\mathbb{D}$ are essentially the shortest curves in $f(\mathbb{D})$. Since the hyperbolic geodesic between 0 and $x \in \mathbb{D}$ is the radial segment $[0, x]$, we state the following version of their theorem.

Gehring-Hayman Theorem. There is a universal constant $C$ with the following property. Suppose $f: \mathbb{D} \rightarrow \mathbb{C}$ is conformal and $\gamma$ is a curve in $\mathbb{D}$ with endpoints 0 and $x \in \mathbb{D}$. Then

$$
\begin{aligned}
& \text { length }(f([0, x])) \leq C \text { length }(f(\gamma)) \\
& \text { 4. } H_{I}^{\psi}=H^{\psi} \text { WHEN } \psi \text { IS DOUBLING }
\end{aligned}
$$

In this section we prove our main theorem, showing that if $\psi$ is a doubling growth function then $H^{\psi}$ and $H_{I}^{\psi}$ contain the same conformal mappings. The main work is done in Lemma 4.3, but first we need the following results involving the nontangential maximal functions $f^{*}$ and $f_{I}^{*}$. We handle the classical setting first.

Lemma 4.1. Let $\psi$ be a growth function and $f: \mathbb{D} \rightarrow \mathbb{C}$ a conformal mapping. If there exists $\delta>0$ such that

$$
\int_{\partial \mathbb{D}} \psi(\delta|f(\omega)|) d \sigma<\infty
$$


then there is $\epsilon(\delta)>0$ such that

$$
\int_{\partial \mathbb{D}} \psi\left(\epsilon f^{*}(\omega)\right) d \sigma<\infty
$$

Proof. First assume that $f(x) \neq 0$ for all $x \in \mathbb{D}$ and let $\phi=\psi^{1 / 2}$. By Lemma 3.5, there exists an absolute constant $C$ such that

$$
\sigma\left(\left\{\omega \in S_{x}: \phi(\delta|f(\omega)|) \geq \phi(\delta|f(x)| / C)\right\}\right) \geq \sigma\left(S_{x}\right) / 2
$$

for every $x \in \mathbb{D}$. Then given any $x \in \mathbb{D}$ we have

$$
\begin{aligned}
\int_{S_{x}} \phi(\delta|f(\omega)|) d \sigma & \geq \phi(\delta|f(x)| / C) \sigma\left(\left\{\omega \in S_{x}: \phi(\delta|f(\omega)|) \geq \phi(\delta|f(x)| / C)\right\}\right) \\
& \geq \phi(\delta|f(x)| / C) \frac{\sigma\left(S_{x}\right)}{2} .
\end{aligned}
$$

Let $M$ denote the non-centered Hardy-Littlewood maximal function on $\partial \mathbb{D}$. By the previous inequality

$$
\phi\left(\delta f^{*}(\omega) / C\right) \leq 2 M \phi(\delta|f|)(\omega),
$$

and so recalling that $M$ is a bounded operator on $L^{2}(\partial \mathbb{D})$ (c.f. [9]) we now have

$$
\begin{array}{r}
\int_{\partial \mathbb{D}} \psi\left(\delta f^{*}(\omega) / C\right) d \sigma=\int_{\partial \mathbb{D}} \phi^{2}\left(\delta f^{*}(\omega) / C\right) d \sigma \leq 4 \int_{\partial \mathbb{D}} M^{2} \phi(\delta|f|)(\omega) d \sigma \\
\leq C_{1} \int_{\partial \mathbb{D}} \phi^{2}(\delta|f(\omega)|) d \sigma=C_{1} \int_{\partial \mathbb{D}} \psi(\delta|f(\omega)|) d \sigma<\infty .
\end{array}
$$

This completes the proof for the case that $f(x) \neq 0$ on $\mathbb{D}$. The other case is handled easily by applying the above result to $g(x)=f(x)-y$, where $y$ is some fixed point in $\mathbb{C} \backslash f(\mathbb{D})$.

The intrinsic version of the lemma can be stated in a nicer form than in the classical setting, since there is no need to consider separate cases.

Lemma 4.2. There exists a universal constant $C$ such that if $\psi$ is a growth function, $f$ is a conformal mapping of $\mathbb{D}$ and $\delta>0$ then

$$
\int_{\partial \mathbb{D}} \psi\left(\frac{\delta}{C} f_{I}^{*}(\omega)\right) d \sigma \leq \int_{\partial \mathbb{D}} \psi\left(\delta|f(\omega)|_{I}\right) d \sigma
$$

Proof. The Gehring-Hayman theorem and (3.2) imply that there is a universal constant $C$ such that

$$
|f(x)|_{I} \leq|f(t \omega)|_{I}+d_{I}(f(t \omega), f(x)) \leq C \text { length }(f([0, \omega))) \leq C|f(\omega)|_{I}
$$

for every $\omega \in \partial \mathbb{D}$ and all $x \in \Gamma(\omega)$. The desired inequality follows.

Given a growth function $\psi$, if there exists a constant $C$ such that $\psi(2 t) \leq C \psi(t)$ for all $t \in[0, \infty]$ then $\psi$ is called doubling. We refer to the infimum of all such $C$ as the doubling constant of $\psi$.

Lemma 4.3. Let $\psi$ be a doubling growth function and $f$ a conformal mapping of $\mathbb{D}$. If there is a map $v: \partial \mathbb{D} \rightarrow[0, \infty]$ such that $\psi(v(\omega)) \in L^{1}(\partial \mathbb{D})$ and

$$
\sup _{x \in \Gamma(\omega)} d(f(x), \partial f(\mathbb{D})) \leq C v(\omega)
$$

for some constant $C$ and almost every $\omega \in \partial \mathbb{D}$ then $f \in H_{I}^{\psi}$. 
Proof. Set $U(\lambda)=\left\{\omega \in \partial \mathbb{D}: f_{I}^{*}(\omega)>\lambda\right\}$. We can use the generalized form of the Whitney decomposition [5, Theorem III.1.3] to write $U(\lambda)$ as the union of caps

$$
U(\lambda)=\cup S_{x_{j}},
$$

where the caps have uniformly bounded overlap and there is an absolute constant $C$ such that for all $j$

$$
\frac{\left(1-\left|x_{j}\right|\right)}{C} \leq d\left(S_{x_{j}}, \partial U(\lambda)\right) \leq C\left(1-\left|x_{j}\right|\right) .
$$

Suppose $\omega \in S_{x_{j}}$ and $v(\omega) \leq \gamma$. Then the initial assumption along with inequalities (3.3) and (4.1) imply that there is $\omega^{\prime} \in \partial \mathbb{D} \backslash U(\lambda)$ and a corresponding $x_{j}^{\prime} \in \Gamma\left(\omega^{\prime}\right)$ such that

$$
\left|f\left(x_{j}\right)\right|_{I} \leq d_{I}\left(f\left(x_{j}\right), f\left(x_{j}^{\prime}\right)\right)+\left|f\left(x_{j}^{\prime}\right)\right|_{I} \leq C \gamma+\lambda .
$$

Let $M>1, \gamma=\frac{\lambda}{(M+1) C}$ and suppose $\omega \in S_{x_{j}}$ with $v(\omega) \leq \gamma$ and $|f(\omega)|_{I}>2 \lambda$. Then (4.2) gives

$$
d_{I}\left(f(\omega), f\left(x_{j}\right)\right) \geq|f(\omega)|_{I}-\left|f\left(x_{j}\right)\right|_{I}>M C \gamma \geq M d\left(f\left(x_{j}\right), \partial f(\mathbb{D})\right),
$$

and so by Lemma 3.4

$$
\begin{gathered}
\sigma\left(\left\{\omega \in S_{x_{j}}:|f(\omega)|_{I}>2 \lambda \text { and } v(\omega) \leq \gamma\right\}\right) \\
\leq \sigma\left(\left\{\omega \in S_{x_{j}}: d_{I}\left(f(\omega), f\left(x_{j}\right)\right)>\operatorname{Md}\left(f\left(x_{j}\right), \partial f(\mathbb{D})\right)\right\}\right) \\
\leq C \sigma\left(S_{x_{j}}\right)(\log M)^{-1}
\end{gathered}
$$

where $C$ is an absolute constant.

If $|f(\omega)|_{I}>2 \lambda$ then also $f_{I}^{*}(\omega)>\lambda$, and so we can apply the above to conclude that

$$
\begin{gathered}
\sigma\left(\left\{\omega \in \partial \mathbb{D}:|f(\omega)|_{I}>2 \lambda\right\}\right) \\
\leq \sigma\left(\left\{\omega \in U(\lambda):|f(\omega)|_{I}>2 \lambda \text { and } v(\omega) \leq \gamma\right\}\right)+\sigma(\{\omega \in \partial \mathbb{D}: v(\omega)>\gamma\}) \\
\leq C \sum_{j} \sigma\left(S_{x_{j}}\right)(\log M)^{-1}+\sigma(\{\omega \in \partial \mathbb{D}: v(\omega)>\gamma\}) \\
\leq C \sigma(U(\lambda))(\log M)^{-1}+\sigma(\{\omega \in \partial \mathbb{D}: v(\omega)>\gamma\}) .
\end{gathered}
$$

Thus

$$
\begin{array}{r}
\int_{\partial \mathbb{D}} \psi\left(\frac{1}{2}|f(\omega)|_{I}\right) d \sigma=\int_{0}^{\infty} \psi^{\prime}(\lambda) \sigma\left(\left\{\omega \in \partial \mathbb{D}:|f(\omega)|_{I}>2 \lambda\right\}\right) d \lambda \\
\leq \int_{0}^{\infty} \psi^{\prime}(\lambda)\left(C \sigma(U(\lambda))(\log M)^{-1}+\sigma\left(\left\{\omega \in \partial \mathbb{D}: v(\omega)>\frac{\lambda}{(M+1) C}\right\}\right)\right) d \lambda \\
=C(\log M)^{-1} \int_{\partial \mathbb{D}} \psi\left(f_{I}^{*}(\omega)\right) d \sigma+\int_{\partial \mathbb{D}} \psi((M+1) C v(\omega)) d \sigma \\
\leq C(\log M)^{-1} \int_{\partial \mathbb{D}} \psi\left(f_{I}^{*}(\omega)\right) d \sigma+C\left(M, C_{\psi}\right) \int_{\partial \mathbb{D}} \psi(v(\omega)) d \sigma
\end{array}
$$

where $C_{\psi}$ denotes the doubling constant of $\psi$.

To finish the proof note that we can apply the above to the functions $f_{t}(x)=f(t x)$ for each $0<t<1$ :

$$
\int_{\partial \mathbb{D}} \psi\left(\frac{1}{2}\left|f_{t}(\omega)\right|_{I}\right) d \sigma \leq \frac{C}{\log M} \int_{\partial \mathbb{D}} \psi\left(f_{t_{I}}^{*}(\omega)\right) d \sigma+C\left(M, C_{\psi}\right) \int_{\partial \mathbb{D}} \psi(v(\omega)) d \sigma .
$$


Then by Lemma 4.2 there is a new absolute constant $C=C\left(C_{\psi}\right)$ such that

$$
\int_{\partial \mathbb{D}} \psi\left(\frac{1}{2}\left|f_{t}(\omega)\right|_{I}\right) d \sigma \leq \frac{C}{\log M} \int_{\partial \mathbb{D}} \psi\left(\frac{1}{2}\left|f_{t}(\omega)\right|_{I}\right) d \sigma+C\left(M, C_{\psi}\right) \int_{\partial \mathbb{D}} \psi(v(\omega)) d \sigma .
$$

By choosing $M$ sufficiently large and combining terms we obtain that

$$
\int_{\partial \mathbb{D}} \psi\left(|f(t \omega)|_{I}\right) d \sigma \leq C \int_{\partial \mathbb{D}} \psi(v(\omega)) d \sigma
$$

for each $0<t<1$, which completes the proof.

The proof of Theorem 1.1 now follows easily.

Proof of Theorem 1.1. If $f \in H^{\psi}$ then $\psi(f(\omega)), \psi\left(f^{*}(\omega)\right) \in L^{1}(\partial \mathbb{D})$ by Fatou's Lemma and Lemma 4.1. If $0 \in \mathbb{C} \backslash f(\mathbb{D})$ then

$$
\sup _{x \in \Gamma(\omega)} d\left(f(x), \partial f(\mathbb{D}) \leq f^{*}(\omega)\right.
$$

for every $\omega \in \partial \mathbb{D}$, and thus $f \in H_{I}^{\psi}$ by Lemma 4.3. The case when $0 \in f(\mathbb{D})$ follows by first applying the result to $g(x)=f(x)-y$, where $y$ is some fixed point in $\mathbb{C} \backslash f(\mathbb{D})$.

Proof of Corollary 1.2. If $f \in H^{\psi}$ then $f \in H_{I}^{\psi}$ by Theorem 1.1, and then by Fatou's Lemma

$$
\int_{\partial \mathbb{D}} \psi\left(|f(\omega)|_{I}\right) d \sigma<\infty
$$

The Gehring-Hayman Theorem now implies that

$$
\int_{\partial \mathbb{D}} \psi\left(\int_{0}^{1}\left|f^{\prime}(r \omega)\right| d r\right) d \sigma<\infty .
$$

Conversely if (4.4) holds, then clearly (4.3) holds by definition. It follows by Lemma 4.1 that $f \in H^{\psi}$.

\section{Counterexample For NON-Doubling $\psi$}

In this section we sketch an example to show that the statement of Theorem 1.1 does not necessarily hold for non-doubling growth functions. The general idea in our example is to use the (quasi-)hyperbolic metric and its (quasi-)invariance to calculate the difference in the growth orders between the maximum modulus

$$
M(r, f)=\sup _{|x|=r}|f(x)|
$$

and the intrinsic maximum modulus

$$
M_{I}(r, f)=\sup _{|x|=r}|f(x)|_{I},
$$

where $f$ maps onto a domain that spirals in a way to maximize $M_{I}(r, f)$. Then, using the maximum moduli we can show that $f \in H^{\psi}$ but $f \notin H_{I}^{\psi}$ for an appropriately chosen growth function $\psi$.

Recall that the quasi-hyperbolic metric on a domain $\Omega$ is defined as

$$
k(u, v)=\inf \int_{\gamma} \frac{1}{d(z, \partial \Omega)}|d z|, \quad u, v \in \Omega,
$$




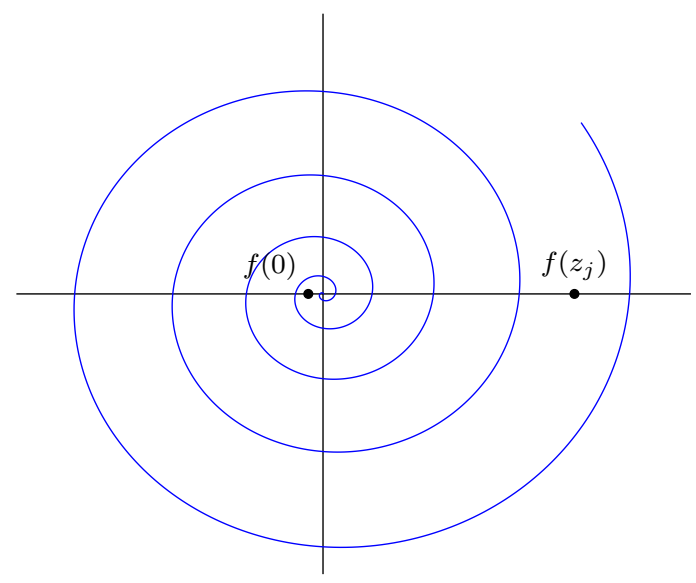

FiguRE 1.

where the infimum is taken over all curves $\gamma \subset \Omega$ with endpoints $u, v$. This metric is quasi-invariant with the hyperbolic metric, and so whenever $f$ is a conformal mapping of $\mathbb{D}$ onto $\Omega$ we have that

$$
\log \left(\frac{1}{1-\left|f^{-1}(u)\right|}\right) \approx k(0, u)
$$

Let $\alpha \geq 0$ be fixed and $g(t)=t^{\alpha+1} e^{2 \pi i t}, t \geq 0$, be the planar spiral curve whose distance between its $j-1$ and $j$ loops is comparable to $j^{\alpha}$. Let $\Omega$ be the simply connected domain having $g(t)$ as its boundary, and let $f$ map $\mathbb{D}$ conformally onto $\Omega$, with $f(0)$ in the center of the spiral. Label as $c_{j}, j \in \mathbb{N}$, the center points of each complete jth loop in $\Omega$, and choose $z_{j} \in \mathbb{D}$ so that $f\left(z_{j}\right)=c_{j}$. See Figure 1 .

It is easy to calculate that

$$
\left|f\left(z_{j}\right)\right| \approx j^{\alpha+1}, \quad\left|f\left(z_{j}\right)\right|_{I} \approx j^{\alpha+2}, \quad \text { and } k\left(0, f\left(z_{j}\right)\right) \approx j^{2} .
$$

Then by (5.1)

$$
\left|f\left(z_{j}\right)\right| \approx\left(\log \frac{1}{1-\left|z_{j}\right|}\right)^{\frac{1+\alpha}{2}}
$$

and

$$
\left|f\left(z_{j}\right)\right|_{I} \approx\left|f\left(z_{j}\right)\right|\left(\log \frac{1}{1-\left|z_{j}\right|}\right)^{\frac{1}{2}}
$$

It easily follows that

$$
M(r, f) \approx\left(\log \frac{1}{1-r}\right)^{\frac{1+\alpha}{2}} \text { and } M_{I}(r, f) \approx\left(\log \frac{1}{1-r}\right)^{\frac{2+\alpha}{2}} .
$$

If $\psi(t)=\exp \left(t^{\frac{2}{1+\alpha}}\right)-1$ then $\psi$ is a growth function that is not doubling. By a theorem in [2], $f \in H^{\psi}$ if and only if there exists $\delta>0$ such that $\int_{0}^{1} \psi(\delta M(r, f)) d r<\infty$. This integral converges in our example by choosing a sufficiently small $\delta$, and so $f \in H^{\psi}$.

For the intrinsic case (3.3) implies

$$
\int_{\partial \mathbb{D}} \psi\left(\delta|f(r \omega)|_{I}\right) d \sigma \gtrsim(1-r) \psi\left(\delta M_{I}(r, f)\right) .
$$


Thus by the previous calculations

$$
\int_{\partial \mathbb{D}} \psi\left(\delta|f(r \omega)|_{I}\right) d \sigma \gtrsim(1-r) \exp \left(C \delta\left(\log \frac{1}{1-r}\right)^{\frac{2+\alpha}{1+\alpha}}\right),
$$

which diverges as $r \rightarrow 1$ no matter the chosen $\delta$, and so $f \notin H_{I}^{\psi}$.

\section{REFERENCES}

[1] K. Astala and P. Koskela. $H^{p}$-theory for quasiconformal maps. Pure Appl. Math. Q., 7(1):19 - 50, 2011.

[2] S. Benedict. Characterizations of Hardy-Orlicz spaces of quasiconformal mappings. To appear.

[3] A. Bonami and S. Grellier. Hankel operators and weak factorization for Hardy-Orlicz spaces. Colloq. Math., 118(1):107-132, 2010.

[4] M. Bonk, P. Koskela, and S. Rohde. Conformal metrics on the unit ball in euclidean space. Proc. London Math. Soc., 3(77):635 - 664, 1998.

[5] R.R. Coifman and G. Weiss. Analyse Harmonique Non-Commutative sur Certains Espaces Homogénes - Lecture Notes in Mathematics, Vol.242,. Academic Press, New York, 1971.

[6] W. Deeb, R. Khalil, and M. Marzuq. Isometric multiplication of Hardy-Orlicz spaces. Bull. Austral. Math. Soc., 34:177 - 189, 1986.

[7] W. Deeb and M. Marzuq. H( $\psi)$ spaces. Canad. Math. Bull., 29(3):295 - 301, 1986.

[8] P. Duren. Theory of $H^{p}$ spaces. Academic Press, Inc., 1970.

[9] J. Garnett. Bounded analytic functions. Academic Press, New York, 1981.

[10] F.W. Gehring and W.K. Hayman. An inequality in the theory of conformal mapping. J. Math. Pures Appl., 41(9):353-361, 1962.

[11] R. Khalil. Inclusions of Hardy Orlicz spaces. Internat. J. Math. and Math. Sci., 9(3):429 - 434, 1986.

[12] Ch. Pommerenke. Boundary Behaviour of Conformal Maps. Springer, 1992.

[13] B.F. Sehba. p-Carleson measures for a class of Hardy-Orlicz spaces. Int. J. Math. Math. Sci., 2011, 2011.

[14] J. Väisälä. Lectures on n-Dimensional Quasiconformal Mappings. Springer-Verlag Berlin., 1971.

[15] M. Zinsmeister. A distortion theorem for quasiconformal mappings. Bull. Soc. Math. France, 114:123 $-133,1986$.

Pekka Koskela \& Sita Benedict

Department of Mathematics and Statistics

University of Jyväskylä

P.O. Box 35 (MaD)

FI-40014

Finland

E-mail addresses: pkoskela@maths.jyu.fi, sita.c.benedict@jyu.fi 